\title{
Clearance of Apoptotic Photoreceptors
}

\section{Elimination of Apoptotic Debris into the Subretinal Space and Macrophage-Mediated Phagocytosis via Phosphatidylserine Receptor and Integrin $\alpha v \beta 3$}

\author{
Toshio Hisatomi, ${ }^{*}$ Taiji Sakamoto, ${ }^{\dagger}$ \\ Koh-hei Sonoda, ${ }^{*}$ Chikako Tsutsumi, ${ }^{*}$ Hong Qiao, ${ }^{*}$ \\ Hiroshi Enaida, ${ }^{*}$ Ichiro Yamanaka, ${ }^{*}$ \\ Toshiaki Kubota, ${ }^{*}$ Tatsuro Ishibashi, ${ }^{*}$ \\ Shinobu Kura, ${ }^{\ddagger}$ Santos A. Susin, ${ }^{\S}$ and \\ Guido Kroemer ${ }^{\text {Tा }}$

\begin{abstract}
From the Departments of Ophthalmology* and Medical Biophysics and Radiation Biology, ${ }^{\ddagger}$ Graduate School of Medical Ophthalmology, ${ }^{\dagger}$ Kagoshima University School of Medicine, Kagoshima, Japan; Apoptosis and Immune System, ${ }^{\S}$ Centre National de la Recherche Scientifique, Institut Pasteur, Paris, France; and the Centre National de la Recherche Scientifique, Institut Gustave Roussy, Villejuif, France
\end{abstract} \\ Sciences, Kyushu University, Fukuoka, Japan; the Department of
}

The effective phagocytotic clearance of apoptotic debris is fundamental to the maintenance of neural tissues during apoptosis. Retinal photoreceptors undergo apoptosis after retinal detachment. Although their induction phase of apoptosis has been well discussed, their phagocytotic process remains quite unclear. We herein demonstrate that apoptotic photoreceptors are selectively eliminated from their physiological localization, the outer nuclear layer, to the subretinal space, and then phagocytosed by monocyte-derived macrophages. This could be shown by an ultrastructural and immunophenotypic analysis. Moreover, in chimera mice expressing transgenic green fluorescent protein in bone marrow-derived cells, the local infiltration of macrophages could be detected after retinal detachment-induced photoreceptor apoptosis. The local injection of an antibody blocking the phosphatidylserine receptor (PSR) or a peptide (GRGDSP)-blocking integrin $\alpha v \beta 3$ revealed that phagocytotic clearance involves the PSR as well as integrin $\alpha v \beta 3$ in vivo. Importantly, the level of blockade obtained with these reagents was different. Although anti-PSR increased the frequency of apoptotic cells that fail to bind to macrophages, GRGDSP prevented the engulfment (but not the recognition) of apoptotic photoreceptor cells by macrophages. To our knowledge, this is the first report describing the mechanisms through which apoptotic photoreceptors are selectively eliminated via a directional process in the subretinal space. (Am J Patbol 2003, 162:1869-1879)

The effective phagocytotic clearance of apoptotic debris is fundamental to the maintenance of neural tissues. The rapid recognition and engulfment of intact apoptotic cells by phagocytes play an important role in preventing secondary necrosis with the consequent release of proinflammatory intracellular products as well as potentially immunogenic self-antigens. In the developmental process and various neural pathologies, although large populations of neurons undergo apoptosis, only few degenerating neurons are observed at a given time within the tissue. ${ }^{1-3}$ This is a consequence of the rapid phagocytosis of apoptotic cells by phagocytes before cell lysis because of secondary necrosis. ${ }^{2}$

Cells dying from apoptosis attract phagocytes, presenting as so-called "eat me" signals, probably by exposing or releasing ligands for receptors on phagocytes. Apoptotic cells develop numerous changes including the exposure of phosphatidylserine (PS) and the alteration of membrane carbohydrates on the cell membrane surface. Several receptors are implicated in the phagocytotic uptake of apoptotic cells by phagocytes, such as phosphatidylserine receptor (PSR), ${ }^{4,5}$ the vitronectin receptor $\left(\alpha \vee \beta 3\right.$ and $\alpha \vee \beta 5$ integrins), ${ }^{6-8}$ CD14, ${ }^{9}$ CD36, ${ }^{10,11}$ or the scavenger receptor class $A .^{12}$ The ligand and receptor systems have been well investigated in vitro. However, both their functions and relative importance remain to be established in vivo. ${ }^{13-17}$

The retina is a highly differentiated neural tissue for vision and exhibits characteristic features of neural apo-

\footnotetext{
Supported in part by grants-in-aid for Scientific Research from the Ministry of Education, Science, Sports, and Culture of the Japanese Government (nos. 09671804 and 09470382) and the Japan National Society for the Prevention of Blindness (Tokyo)

Accepted for publication February 26, 2003

Address reprint requests to Dr. Toshio Hisatomi, M.D., Ph.D., Department of Ophthalmology, Graduate school of Medical Sciences, Kyushu University, 3-1-1 Maidashi, Higashi-ku, Fukuoka, 812-8582, Japan. E-mail: hisatomi@med.kyushu-u.ac.jp.
} 
ptosis in various pathologies. As a result, retinal detachment (RD) causes the selective apoptotic demise of retinal photoreceptors. The induction phase of photoreceptor apoptosis in RD has been characterized to some extent, ${ }^{1,18,19}$ establishing that it involves a caspase-independent apoptotic process ${ }^{3}$ that can be inhibited by the intraocular injection of brain-derived neurotrophic factor (BDNF). ${ }^{3,20}$ Importantly, the inhibition of apoptosis allows for the maintenance of the retinal function and the prevention of RD-induced blindness. ${ }^{21}$ Although the induction phase of photoreceptor apoptosis in RD has been previously reported, ${ }^{1,3,18,21}$ their clearance phases are still unknown. Identifying the phagocytotic process, including the infiltrating phagocytes, their distribution and recognition systems for apoptotic photoreceptors thus remain an obviously important issue to prevent tissue destruction by their released contents. The destruction of these tissues causes retinal gliosis, and an excessive amount of gliosis is sometimes harmful for the maintenance of retinal regular structure and function. ${ }^{22}$ Previous studies have identified candidate phagocytes of retinal apoptotic cells in the developmental or pathological processes, ${ }^{23-26}$ without, however, providing any insight into the mechanism of these processes. Apoptotic photoreceptors have thus been thought to be phagocytosed in the original location of the outer nuclear layer by a diverse range of cells including retinal pigment epithelium (RPE), retinal microglia, infiltrated macrophages, tissue macrophages, glial cells, or neighboring cells. ${ }^{27-29}$

To elucidate the mechanisms accounting for the elimination of apoptotic photoreceptors, we used a rodent model of RD-induced retinal degeneration. ${ }^{3,21}$ Unexpectedly, we found that apoptotic photoreceptors are eliminated from their original location, the outer nuclear layer, and thereafter move into the subretinal space, where they are phagocytosed by infiltrating macrophages derived from freshly recruited circulating monocytes. In addition, we also report that the PSR and the integrin $\alpha \vee \beta 3$ play an instrumental role in the phagocytotic recognition and internalization of apoptotic photoreceptors in vivo.

\section{Materials and Methods}

\section{Experimental $R D$}

All procedures conformed to the Association for Research in Vision and Ophthalmology's statement for the Use of Animals in Ophthalmic and Vision Research. Brown Norway rats (Kyudo, Fukuoka, Japan), postnatal 8 weeks, were studied as follows. Rat experimental RD was produced by a previously described method. ${ }^{3,28}$ Briefly, the rats were anesthetized with an intraperitoneal injection of pentobarbital and their pupils were dilated with topical $1 \%$ tropicamide and $2.5 \%$ phenylephrine hydrochloride. The retinas were detached using a subretinal injection of $1 \%$ sodium hyaluronate (Healon; Pharmacia, Uppsala, Sweden). Sodium hyaluronate $(0.05 \mathrm{ml})$ was gently injected through the sclera into the subretinal space to enlarge the RD. Because the volume of the vitreous space is relatively small, the injection of $0.05 \mathrm{ml}$ sodium hyaluronate reproducibly produced similar RDs (half of the retina). Biomicroscopy (Kowa, Tokyo, Japan) demonstrated that the same portion of the retina remained detached for more than 28 days after treatment. The detached retinas that reattached spontaneously were thus excluded from the further studies. Eyes with an anterior chamber puncture, scleral incision, and no injection of sodium hyaluronate were used as controls. The rats were sacrificed at 6 and 12 hours and on days 1, 3, $5,7,14$, and 28 after treatment and then their eyes were harvested for the following studies.

\section{Chimera Mouse by Transplantation with Green Fluorescent Protein (GFP)-Transgenic Bone Marrow Cells}

Previously, the phagocytes that infiltrated after RD were reported to be RPE, retinal microglia, or monocyte-derived macrophages, however, this identification remains unclear. ${ }^{27,29}$ To characterize the infiltrating phagocytes, we produced chimera GFP mice, by a previously described method. Briefly, we used transgenic mouse lines with an enhanced GFP (EGFP) cDNA under the control of a chicken $\beta$-actin promoter and cytomegalovirus enhancer (a generous gift from Dr. Masaru Okabe, Osaka University, Osaka, Japan). ${ }^{30,31}$ All of the tissues from these transgenic lines, with the exception of erythrocytes and hair were green under excitation light. The established transgenic mouse was used as a cell source for GFP-positive bone marrow cells. The GFP-positive cells were transplanted intravenously in C57/B6 mice after irradiation with 9 Gy of X-rays. The eyes were protected with lead shields for prevention of radiation retinopathy. $A$ successful bone marrow transplantation was confirmed by the identification of GFP-positive cells in the blood 2 weeks after irradiation. RD was produced in these chimera mice as described above. These eyes were harvested 3 days after RD, cryosectioned, acetone-fixed, and then were observed by fluorescent microscopy (Olympus, Tokyo, Japan). The eyes from wild-type (wt) mice were used as control eyes.

\section{Terminal dUTP Nick-End Labeling (TUNEL)}

Apoptotic photoreceptor degeneration was detected by TUNEL. Four- $\mu$ m-thick sections were made from samples fixed in $4 \%$ paraformaldehyde and embedded in paraffin. TUNEL staining was performed with the ApopTag Fluorescein Direct in Situ Apoptosis Detection Kit (Intergen Company, New York, NY) according to the manufacturer's protocol. The sections were co-stained with propidium iodide (Molecular Probes, Eugene, OR), thus allowing the observation of the cell nuclei by a fluorescence microscope (Olympus). Ten sections for each eye specimen were randomly selected and observed by masked observers (six eyes for each time point). The results are presented as the means \pm SD. 


\section{AIF, ED1, and ED2 Immunohistochemistry}

Apoptosis-inducing factor (AIF) is a novel, caspase-independent apoptogenic mediator in apoptosis, which is normally confined to the mitochondrial intermembrane space, yet translocates to the cytosol and the nucleus in the apoptotic process. ${ }^{32}$ In a previous report, we confirmed that the mitochondrio-nuclear relocalization of AIF occurred in this model. ${ }^{3}$ To clarify whether AIF participated in the late phase of apoptosis in the subretinal apoptotic nucleus, we examined this issue immunohistochemically. Samples were fixed in 4\% paraformaldehyde, embedded in paraffin, deparaffinized in xylene, rehydrated in ethanol, and washed in phosphate-buffered saline (PBS), as described above. A 1:100 dilution of anti-AIF rabbit serum was produced by a previously described method ${ }^{32}$ and incubated at $4^{\circ} \mathrm{C}$ overnight. A nonimmune serum and a preabsorbed antiserum (with 1 $\mu \mathrm{g} / \mu \mathrm{l}$ of recombinant AIF) were used as negative controls. To characterize the infiltrating phagocytes, antiED1, ED2 antibodies (Serotec, Oxford, UK), F4/80 antibody (Caltag Laboratories, Burlingame, CA), anti-pan cytokeratin (DAKO, Glostrup, Denmark), and control IgG were used at a 1:100 dilution. Anti-ED1 antibody recognized monocytes/macrophages, anti-ED2 recognizes tissue macrophages in rat, ${ }^{33} \mathrm{~F} 4 / 80$ antibody recognizes mouse macrophages, ${ }^{34}$ anti-pan cytokeratin recognizes RPE. Cy5-labeled secondary antibody (Zymed Laboratories, San Francisco, CA) was used at a dilution of 1:200 for 20 minutes. The sections were co-stained by TUNEL and observed by fluorescence microscopy.

\section{Transmission Electron Microscopy and Immunoelectron Microscopy}

The eyes were enucleated and the posterior segments were fixed in 1\% glutaraldehyde and $1 \%$ paraformaldehyde in PBS. The detached retinas were removed and postfixed in veronal acetate buffer osmium tetroxide $(2 \%)$, dehydrated in ethanol and water, and embedded in Epon. Ultrathin sections were cut from blocks and mounted on copper grids. For immunoelectron microscopy, the eyes were fixed in $1 \%$ paraformaldehyde in PBS, and the detached retinas were rinsed with PBS, incubated in $\mathrm{NH}_{4} \mathrm{Cl}$, and embedded in London Resin white blocks (London Resin, London, UK). Primary antibody for AIF was used at a 1:150 dilution, and the sections were incubated at $4^{\circ} \mathrm{C}$ overnight. A nonimmune serum was used as a negative control. Anti-rabbit antibody conjugated with 10-nm gold particles (British BioCell, Cardiff, UK) was used as a secondary antibody at a dilution of 1:30 for 90 minutes. The specimens were observed with a JEM 100CX electron microscope (JEOL, Tokyo, Japan).

\section{Scanning Electron Microscopy}

The removed retinas were postfixed in veronal acetate buffer osmium tetroxide (2\%), and dehydrated in ethanol and water. The retinas were saturated in $t$-butyl alcohol, and critical point drying was performed (Eiko, Tokyo, Japan). The tissue was then placed on stubs by means of self-adhering carbon tabs and sputtered with $\mathrm{Au}$ of 20-nm thickness by argon plasma coater (Eiko). Next, the subretinal surface of the detached retina was studied by a JEM 840 scanning electron microscope (JEOL).

\section{Inhibition of Phagocyte Infiltration by Preventing Apoptosis Development}

It was reported that BDNF inhibited photoreceptor apoptosis after RD. ${ }^{3,20,21}$ To investigate the relationship between photoreceptor apoptosis and phagocyte infiltration, we administered BDNF (2.5 ng per eye; R\&D Systems, Minneapolis, MN) into the subretinal space. To clarify whether apoptotic photoreceptors attract phagocytes, the phagocyte infiltration into the subretinal space was determined on day 3 . The number of cells per $1-\mathrm{mm}$ retina in 10 histological sections for each eye was measured and six eyes were analyzed using analysis software (MacScope; Mitani, Fukui, Japan). The results were expressed as the means $\pm \mathrm{SD}$.

\section{Blockade of Phagocytotic Process by Inhibiting Receptor Systems}

To investigate the participation of candidate receptors for phagocytosis of apoptotic photoreceptors, we used RGD peptide (Bachem, Bubendorf, Switzerland), anti-integrin $\beta 3$ antibody (clone F11; BD Pharmingen, San Diego, CA) for integrin $\alpha \vee \beta 3$, and anti-PSR antibody (Cascade BioScience, Winchester, MA). The integrin $\alpha \vee \beta 3$ and PSR are shown to be present on monocyte-derived macrophages. ${ }^{10,35}$ The function-blocking hexapeptide GRGDSP including the integrin $\alpha v \beta 3$-binding motif RGD, and nonfunctioning control hexapeptide GRGESP were administered into the subretinal space mixed in $1 \%$ sodium hyaluronate at a concentration of $2.0 \mathrm{mg} / \mathrm{ml}$. Anti-integrin $\beta 3$ antibody, which blocks integrin $\alpha \vee \beta 3$ binding, and control IgG were injected with 1\% sodium hyaluronate at a concentration of $1.0 \mathrm{mg} / \mathrm{ml}$. Anti-PSR antibody, which blocks phosphatidylserine binding, and control IgM were injected at a concentration of $1.0 \mathrm{mg} / \mathrm{ml}$. The eyes were then harvested on day 3 and analyzed by immunohistochemistry, transmission electron microscopy, and scanning electron microscopy. The number of cells per $1-\mathrm{mm}$ retina in 10 histological sections for each eye was measured and six eyes were analyzed using analysis software (MacScope). The results were expressed as the means $\pm \mathrm{SD}$. Student's $t$-test was used to calculate the probability by comparing data between the groups and $P<0.05$ was considered to be significant.

\section{Results}

\section{Photoreceptor Apoptosis Detection by TUNEL after $R D$}

After the injection of sodium hyaluronate into the subretinal space of rats, RD was followed macroscopically and 

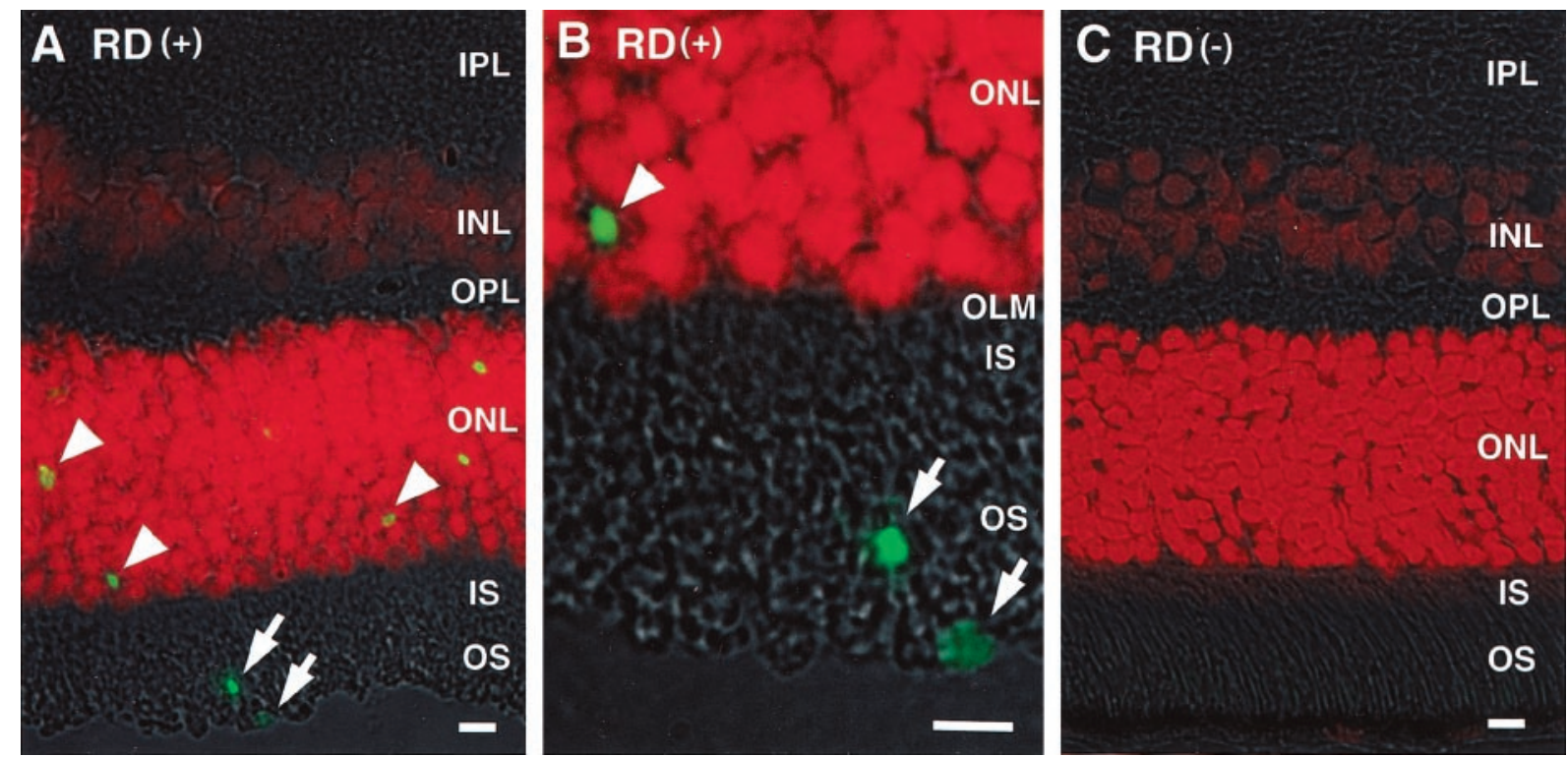

Figure 1. Fluorescent micrographs of photoreceptor apoptosis by TUNEL. Each section was stained by TUNEL (green) and propidium iodide. A: Detached retina showing photoreceptor apoptosis (arrowheads) (IPL, inner plexiform layer; INL, inner nuclear layer; OPL, outer plexiform layer; ONL outer nuclear layer; IS, inner segment; OS, outer segment). B: High-magnification image of the outer retina. TUNEL-positive staining is seen in the outer segment layer and the subretinal space (arrows) (OLM, outer limiting membrane). C: Control retina without RD showed no positive staining for TUNEL. Original magnifications: $\times 200($ B); $\times 600$ (C). Scale bar, $5 \mu \mathrm{m}$

histologically for 28 days. Macroscopically, the detached area remained unchanged during this period. TUNELpositive apoptotic cells appeared mainly in the photoreceptors starting 12 hours after $\mathrm{RD}$, reached a maximum on day 3 (Figure 1, A and B, arrowheads), and then gradually decreased. ${ }^{3}$ Although most TUNEL-positive signals were observed in the outer nuclear layer, some signals were observed in the outer segment layer and subretinal space (Figure 1, arrows). In control eyes without RD, no TUNEL-positive signals were observed in any of the layers (Figure 1C).

\section{Ultrastructural Studies of Apoptotic Photoreceptors and Electron-Dense Materials}

RD-induced apoptotic morphological alterations, including chromatin condensation, cell shrinkage, and apoptotic body formation were observed in the photoreceptors (Figure 2; A to D). In the apoptotic body formation process, nucleolar components are tightly aggregated (Figure 2B), separated into semi-dense granular masses (Figure 2C) and forms electron-dense linear strands (Figure 2D). As electron-dense apoptotic body formation developed, the electron-dense materials became detectable in the inner and outer segment layers and the subretinal space (Figure 2; $\mathrm{E}$ to $\mathrm{G}$ ). Figure 2E shows the electron-dense materials just passing through the outer limiting membrane, which consisted of portions of Müller cells with adherent junctions. These electron-dense materials are apparently mixed with other cytosolic structures such as mitochondria (Figure 2E). The electrondense materials were found among the normal outer segments in outer segment layer (Figure 2, F and G).

\section{AlF Immunohistochemistry}

Apoptotic photoreceptor nuclei stain positively with an antibody specific for the AIF protein, thus indicating the translocation of this protein from the mitochondria to the nucleus (Figure 3, A and B). ${ }^{3}$ As photoreceptor apoptosis developed, TUNEL-positive signals were also detected in the outer segment layer, and these are still positive for AIF (Figure 3A). Immunoelectron microscopy showed the electron-dense materials in the outer segment layer to be AlF-positive by gold particles (Figure 3C). The nonimmune serum and preabsorbed serum showed no positive staining for AIF (data not shown).

\section{Immunophenotypic Analysis of Infiltrating Phagocytes and Phagocytosis of Apoptotic Bodies in the Subretinal Space}

Phagocyte infiltration into the subretinal space occurred 2 days after RD. Most infiltrating phagocytes contained in the subretinal space (88\%) bore the characteristic phenotype of monocyte-derived macrophages (ED1 ${ }^{+}, \mathrm{ED2}^{-}$; Figure 4; A to C) while a minority was consisted of cells bearing the specific phenotype of RPE (pan cytokeratin ${ }^{+}$; Figure 4D). Most if not all ED1 ${ }^{+}$cells were located in the outer segment layer of photoreceptors or the subretinal space of the detached retina (Figure 4A). A low frequency of ED2 ${ }^{+}$cells, namely tissue macrophages, were detected in the inner layers of the detached retina (Figure $4 B$ ), yet were never found to phagocytose apoptotic debris. Double-staining experiments confirmed that ED1 ${ }^{+}$ macrophages phagocytosed $\mathrm{TUNEL}^{+}$apoptotic bodies 

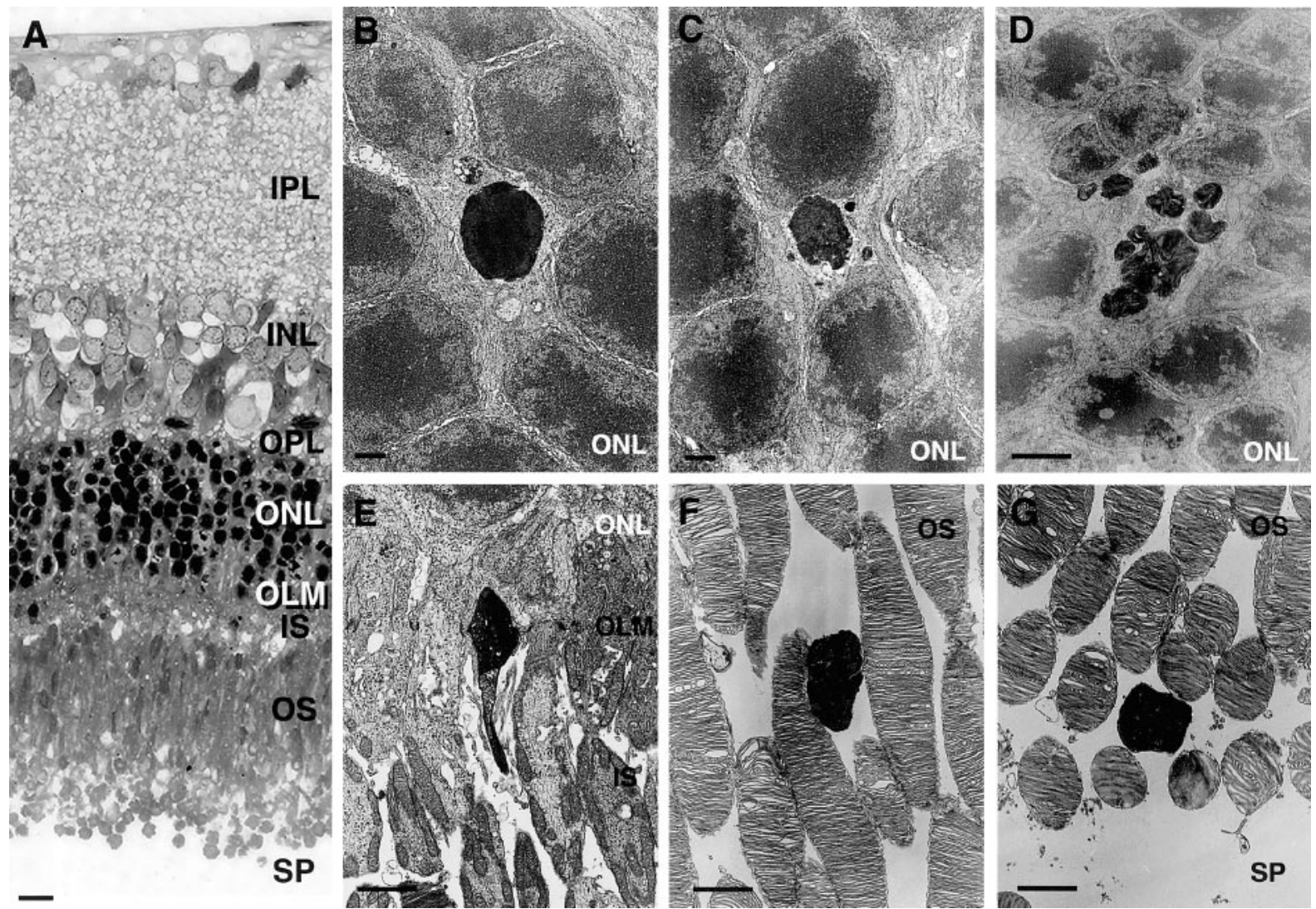

Figure 2. Light (A) and electron (B to G) micrographs of photoreceptor apoptosis. It is not a time-lapse sequence but a series of images selected and arranged to present a probable sequence of events (B to $\mathbf{G})$. Apoptotic photoreceptor shows cell shrinkage and chromatin condensation in ordinary location of outer nuclear layer (B) and apoptotic body formation (C). In the apoptotic body formation process, nucleolar components are tightly aggregated (B), separated into semi-dense granular masses (C), and form electron-dense linear strands (D). As electron-dense apoptotic body formation developed, the electron-dense materials became detectable in the inner and outer segment layers and the subretinal space $(\mathbf{E}-\mathbf{G})$. These electron-dense materials are apparently mixed with other cytosolic structures such as mitochondria $(\mathbf{E})$. The electron-dense materials were found among the normal outer segments in the outer segment laver $(\mathbf{F}$ and $\mathbf{G})$. Original magnifications: $\times 200(\mathbf{A}) ; \times 4000(\mathbf{B}$ and $\mathbf{C}) ; \times 3300(\mathbf{D}-\mathbf{G})$. Scale bars: $10 \mu \mathrm{m}(\mathbf{A}) ; 2 \mu \mathrm{m}(\mathbf{B}$ and $\mathbf{C}) ; 5 \mu \mathrm{m}(\mathbf{D}-\mathbf{G})$
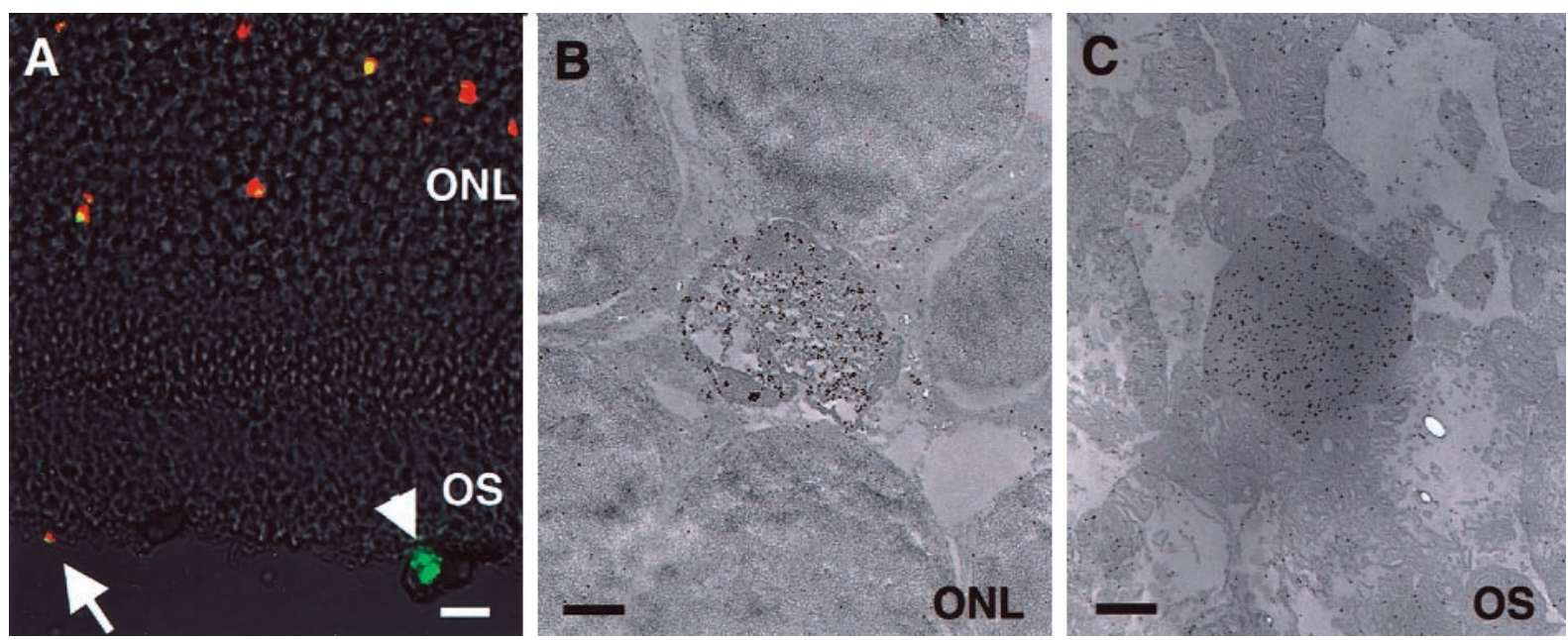

Figure 3. Immunohistochemistry of apoptosis-inducing factor (AIF). A: Fluorescent micrographs of the detached retina, stained with AIF (red) and TUNEL (green). TUNEL-positive apoptotic photoreceptors are also AIF-positive. The eliminated apoptotic photoreceptor debris in the subretinal space is still positive for AIF and TUNEL (arrow). AIF staining is not seen in the TUNEL-positive apoptotic debris phagocytosed by macrophages (arrowhead). B: Immunoelectron micrographs of the detached retina fixed in $1 \%$ paraformaldehyde and embedded in resin. The degenerated apoptotic photoreceptors were AIF-positive, while neighboring normal photoreceptors were negative. C: Eliminated photoreceptor nucleus in outer segment layer still shows AIF-positive staining. Original magnifications: $\times 200(\mathbf{A}) ; \times 5000(\mathbf{B}$ and $\mathbf{C})$. Scale bars: $10 \mu \mathrm{m}(\mathbf{A}) ; 1 \mu \mathrm{m}(\mathbf{B}$ and $\mathbf{C})$. 

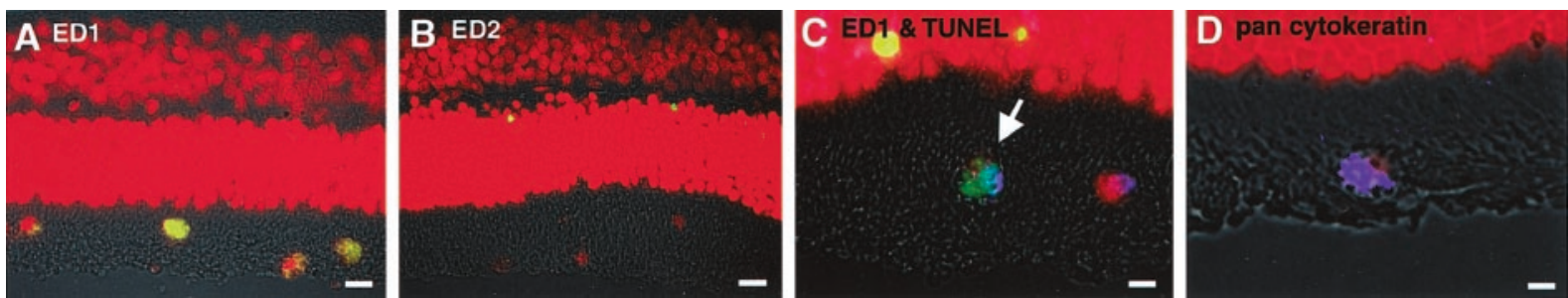

Figure 4. Characterization of infiltrating macrophages by immunohistochemistry, TUNEL (green), and propidium iodide (red). The most infiltrating phagocytes are ED1-positive, monocyte-derived macrophages (A: ED1, yellow). Although the infiltrating phagocytes are ED2-negative, few ED2-positive tissue macrophages are seen in the inner retina (B: ED2, yellow). TUNEL-positive apoptotic photoreceptor debris is phagocytosed by ED1-positive macrophages (C: arrow; ED1, blue TUNEL, green). In the peripheral area of the detached retina, a few pan cytokeratin-positive RPEs were observed (D, blue). Original magnifications: $\times 200$ (A and B); $\times 400(\mathbf{C}$ and $\mathbf{D})$. Scale bars: $10 \mu \mathrm{m}(\mathbf{A}$ and $\mathbf{B}) ; 5 \mu \mathrm{m}(\mathbf{C}$ and $\mathbf{D})$.

(Figure 4C). The free RPE that migrated into the peripheral area of the detached retina were positive for a specific marker, pan cytokeratin (Figure 4D).

\section{GFP-Positive Phagocyte Infiltration after $R D$ in the Chimera Mouse}

To further elucidate the origin of phagocytes, we used chimera mice, reconstituted with bone marrow-expressing transgenic GFP. Although such mice normally lack bone marrow-derived $\left(\mathrm{GFP}^{+}\right)$macrophages in their retina (Figure 5A), they exhibit infiltrating $\mathrm{GFP}^{+}$phagocytes several days after RD (Figure 5B). The infiltrated GFP ${ }^{+}$ phagocytes are positive for mouse macrophage marker F4/80 (Figure 5C). The 84\% of the total infiltrated phagocytes are $\mathrm{GFP}^{+}, \mathrm{F}_{4} / 80^{+}$cells in the chimera mouse. The $\mathrm{GFP}^{+}$cells were never seen in the wild-type control mice after RD (Figure 5D).

\section{Ultrastructural Characterization of Infiltrating Phagocytes by Transmission Electron Microscopy and Scanning Electron Microscopy}

Ultrastructural observations could differentiate two types of infiltrating cells. One is a middle-sized (10 $\mu \mathrm{m}) \mathrm{mac}$ rophage-like cell abundant with lysosomes and fingerlike processes (Figure 6; $A$ to $C$ ), whereas the other is a large $(15 \mu \mathrm{m})$, round cell abundant with melanin granules (Figure 6; D to F). The infiltrating macrophage-like cells phagocytosed apoptotic condensed nuclei rather than degenerated outer segment debris (Figure 6A). These macrophage-like cells apparently infiltrated from choroidal vessels, and migrated through the Bruch's membrane and RPEs toward the subretinal space (Figure 6C). The other melanin-rich round cells also migrated and infiltrated into the subretinal space from the peripheral area of the detached retina (Figure 6; D to F). Early after RD, the macrophage-like cells mostly phagocytosed the apoptotic nuclei (Figure 6A), while later on the pattern of digestion changed. As the apoptotic photoreceptor decreased on days 14 and 28, these cells instead phagocytosed the degenerated outer segment of the photoreceptors (data not shown).

\section{Phagocyte Infiltration Induced by Apoptotic Photoreceptors and Their Debris}

In control retina without RD, both apoptotic photoreceptors and apoptotic debris were rarely seen (Table 1 and Figure 1C). No phagocyte infiltration was noted in subretinal space of control retina (Table 1). RD induced photoreceptor apoptosis as well as phagocyte infiltration in the subretinal space. The subretinal injection of a neurotrophic factor, BDNF, dramatically reduced photoreceptor apopto-
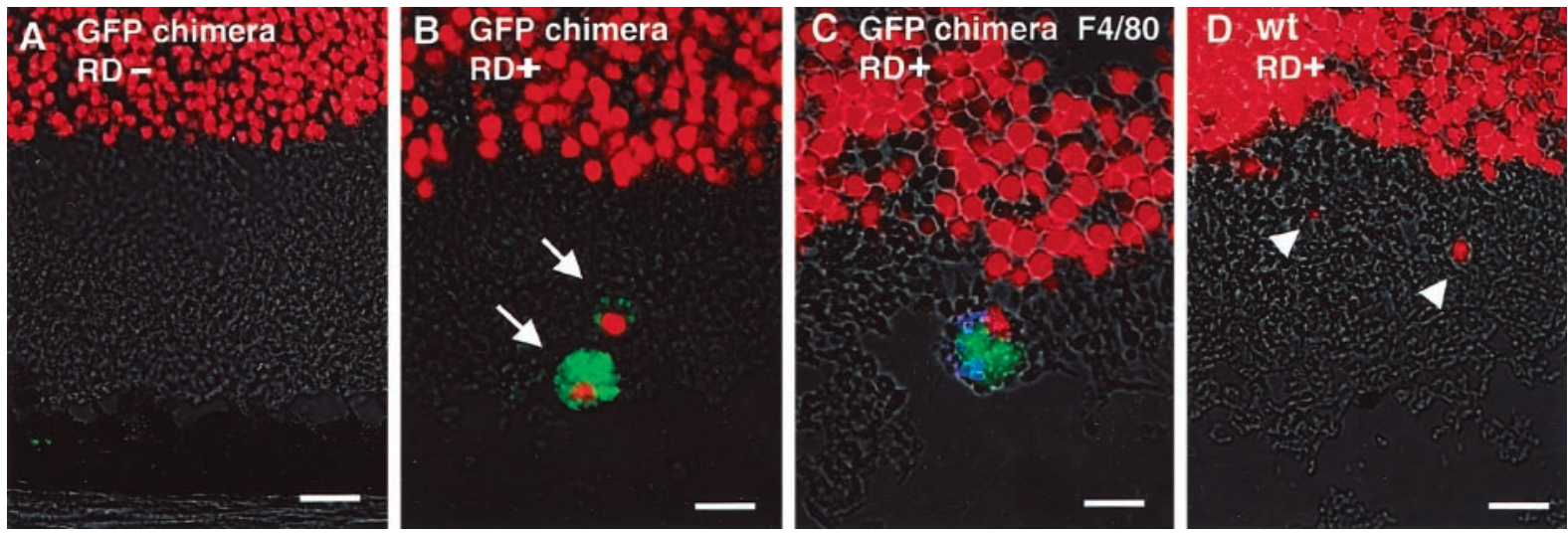

Figure 5. Evidence that infiltrating phagocytes after RD are derived from bone marrow cells with GFP transgenic/wild-type chimera mouse. GFP transgenic mouse was produced, and the bone marrow was used as a cell source of GFP-positive bone marrow cells (see Materials and Methods). Each specimen was stained by propidium iodide to show cell nuclei (red). In GFP chimera mouse, although GFP-positive cells were not observed in attached retina (A), GFP-positive cells dramatically infiltrated into the subretinal space after RD (B, green, arrows). These GFP-positive phagocytes are also positive for F4/80 (C, GFP-green, F4/80-blue). In wild-type (wt) mouse, no GFP-positive cells are observed even after RD (D, arrowheads). Original magnifications, $\times 400$. Scale bar, $10 \mu \mathrm{m}$. 

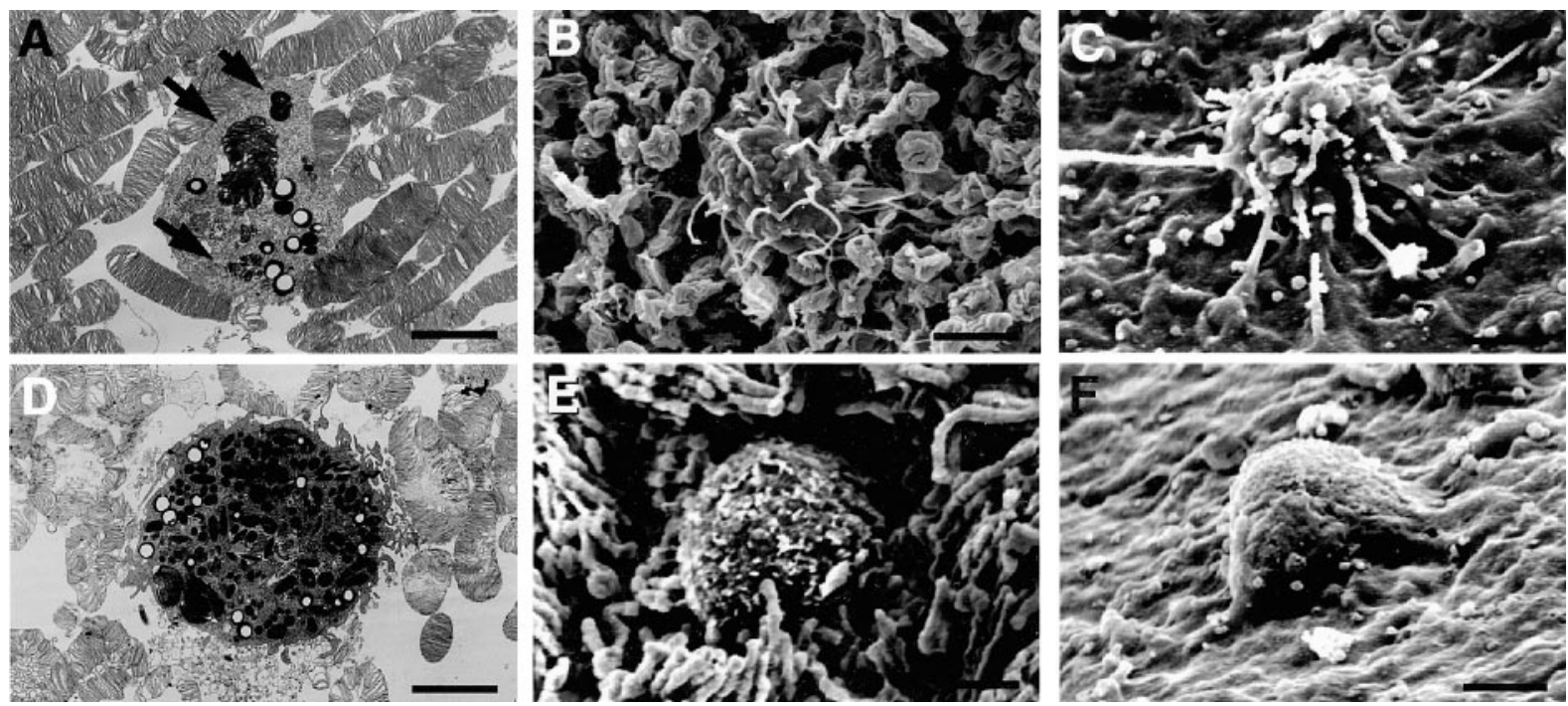

Figure 6. Characterization of infiltrating cells into the subretinal space by ultrastructural studies. Ultrastructural observations could differentiate two types of infiltrating cells. One is a middle-sized $(10 \mu \mathrm{m})$ macrophage-like cell abundant with lysosomes and finger-like processes (A-C), whereas the other is a large (15 $\mu \mathrm{m})$, round cell abundant with melanin granules (D-F). Most infiltrating phagocytes are macrophage-like cells (A and $\mathbf{B}$ ). These cells selectively phagocytose apoptotic photoreceptor debris (A, arrow), elongate their filopodia for the apoptotic debris (B) in the subretinal surface of the detached retina. These cells infiltrate to subretinal space through the RPEs from choroidal vessels $(\mathbf{C})$. In the peripheral area of the detached retina, a small amount of the other large rounc cells migrate and infiltrate into the subretinal space (D and $\mathbf{E}$ ). The large round cells migrated from RPE monolayer beneath the detached retina (F). Original magnifications: $\times 2600(\mathbf{A}, \mathbf{D}) ; \times 2500(\mathbf{B}, \mathbf{C}, \mathbf{E}, \mathbf{F})$. Scale bar, $5 \mu \mathrm{m}$.

sis and phagocyte infiltration into subretinal space (Table 1). In contrast, the injection of anti-PSR antibody, RGD peptide, and anti-integrin $\beta 3$ antibody increased apoptotic debris and phagocyte infiltration (Table 1).

\section{Anti-PSR Antibody Inhibited the Recognition of Apoptotic Debris by Macrophages}

An antibody specific for the PSR, capable of blocking the interaction between PSR and PS, ${ }^{35}$ was administered into the subretinal space when RD was induced. Eyes were harvested 3 days later, and then were fixed and embedded in paraffin. The sections were double-stained by TUNEL and propidium iodide and 10 sections for each eye specimen were randomly selected and observed in a masked manner $(n=6)$. The subretinal apoptotic bodies were classified as free, as bound to macrophages, or as phagocytosed (Figures 7 and 8). Anti-PSR significantly blocked the uptake of apoptotic photoreceptor debris by macrophages (Figures 7 and 8). As a result, the free apoptotic debris increased from 10 per $1-\mathrm{mm}$ retina in controls injected with a control IgM to 17 per 1-mm retina in animals treated with anti-PSR (Figure 8, anti-PSR IgM, $P<0.05)$. These data indicate that the PS-PSR interaction has a rate limiting effect on the macrophage-mediated recognition of apoptotic photoreceptor cells from the subretinal space.

\section{RGD Peptide and Anti-Integrin $\beta 3$ Antibody Inhibition of Internalization of Apoptotic Debris by Macrophages}

The hexapeptide GRGDSP includes the integrin $\alpha \vee \beta 3$ binding motif RGD and thus blocks the interaction be-

Table 1. Cell Counts per 1-mm Width of the Retina

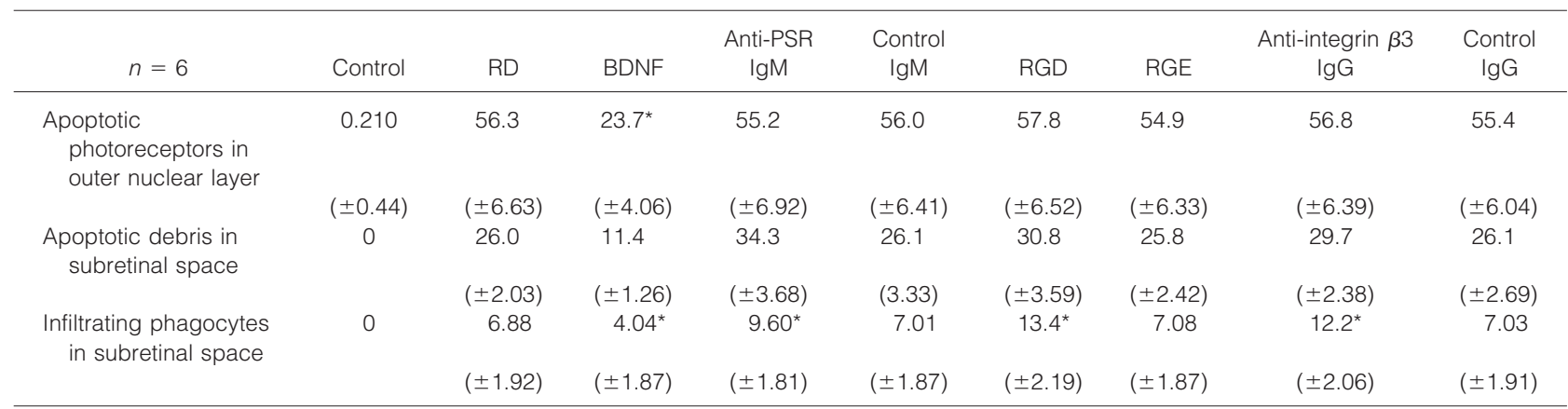

The number of apoptotic photoreceptors in outer nuclear layer and infiltrating phagocytes in the subretinal space. RD was made with subretinal injection of sodium hyaluronate and the eyes were then harvested on day 3 and analyzed by TUNEL and immunohistochemistry ( $n=6$ ). Ten sections for each eye specimen were randomly selected and observed by masked observers. The results are presented as the means \pm SD. BDNF dramatically decreased both photoreceptor apoptosis and phagocyte infiltration $\left({ }^{*}, P<0.05\right)$. In contrast, anti-PSR antibody, RGD peptide, antiintegrin $\beta 3$ antibody increased apoptotic debris and phagocyte infiltration $\left({ }^{*}, P<0.05\right)$. 

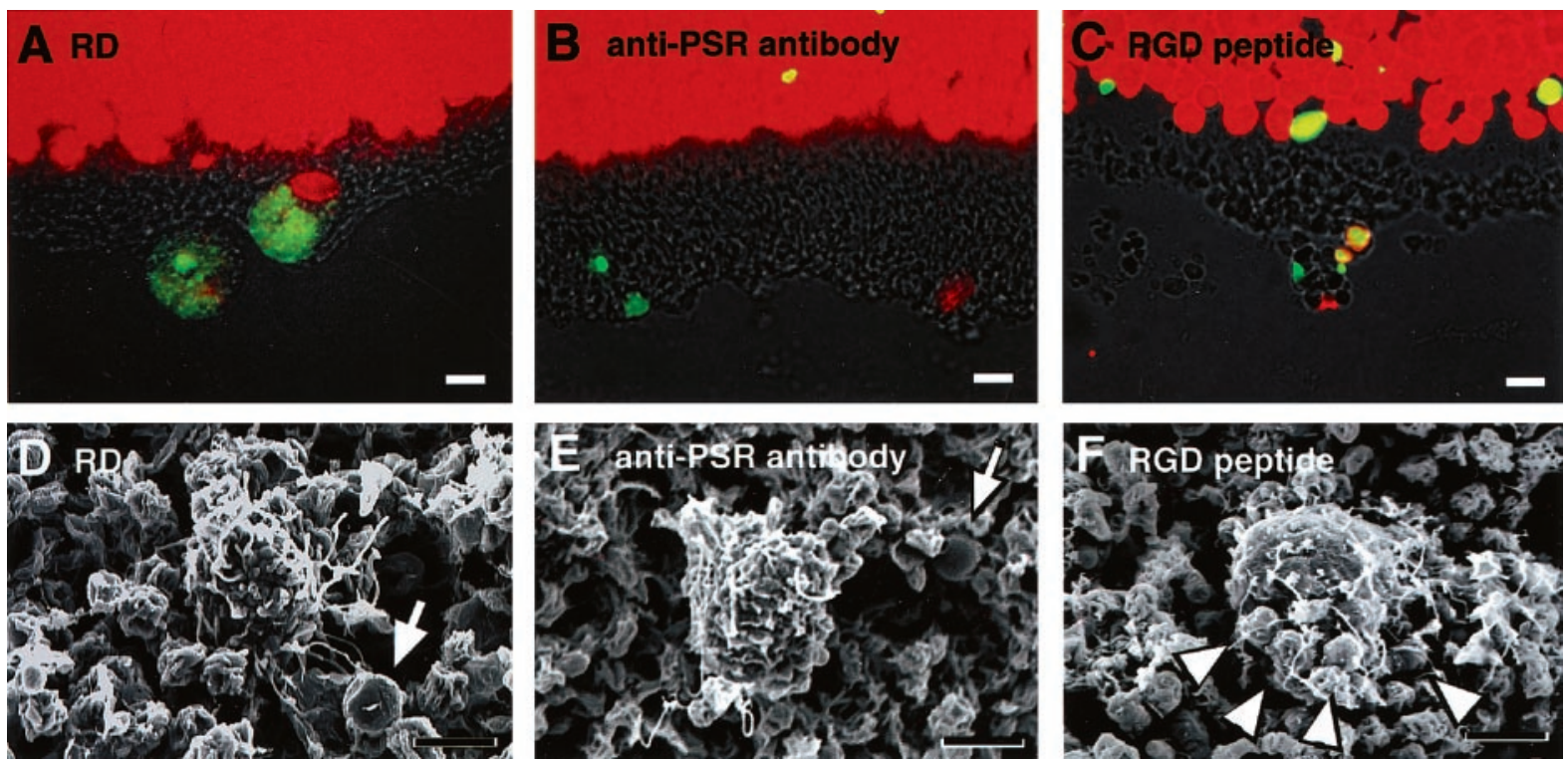

Figure 7. The blockade of the macrophage phagocytosis by inhibiting receptor systems. Fluorescent images (A-C) show apoptotic debris (TUNEL, green), nucleus (propidium iodide, red), and phase contrast images (gray). Scanning electron microscopy revealed eliminated apoptotic photoreceptor debris (arrows) and infiltrating macrophages (D-F). The infiltrating macrophages selectively recognize and phagocytose a large amount of TUNEL-positive apoptotic debris (A green). In contrast, the function-blocking anti-PSR antibody blocks apoptotic debris recognition by infiltrating macrophages (B and $\mathbf{E}$ ). The function-blocking peptide with integrin $\alpha \mathrm{v} \beta 3$-binding motif RGD does not block the recognition and binding of apoptotic debris by macrophages, but blocks internalization of these particles $(\mathbf{C}$, green; and $\mathbf{F})$. The binding of numerous apoptotic bodies to a single macrophage is shown $(\mathbf{F}$, arrowheads). Original magnifications: $\times 400(\mathbf{A}-\mathbf{C})$; $\times 2500($ D-F). Scale bars, $5 \mu \mathrm{m}$.

tween $\alpha v \beta 3$ and $\alpha v \beta 3$ ligands such as vitronectin. ${ }^{10}$ The local injection of this peptide blocked macrophage phagocytosis of apoptotic photoreceptors (Figures 7 and 8). Although the infiltrated macrophages recognized and collected apoptotic debris, which apparently bound to their surface, subsequent internalization was blocked by GRGDSP (Figures 7 and 8). Scanning electron microscopy revealed that macrophages selectively recognized and collected apoptotic debris yet failed to engulf apoptotic bodies (Figure 7F). As an internal control, the peptide GRGESP (which lacks the RGD sequence) did not block the phagocytotic uptake of apoptotic debris, and never caused an increase of bound apoptotic debris on the infiltrated phagocytes (Figure 8). The results were confirmed by the antibody clone F11 (anti-integrin $\beta 3$ antibody), which blocks the integrin $\alpha \vee \beta 3$. This antibody also blocked the internalization of apoptotic bodies by phagocytes, thus resulting in an increased amount of bound apoptotic debris and infiltrating phagocytes (Figure 8 and Table 1). These data underscore the importance of an interaction between integrin $\alpha \mathrm{v} \beta 3$ and an RGD motif-containing $\alpha \vee \beta 3$ ligand for the engulfment of apoptotic cells in our model.

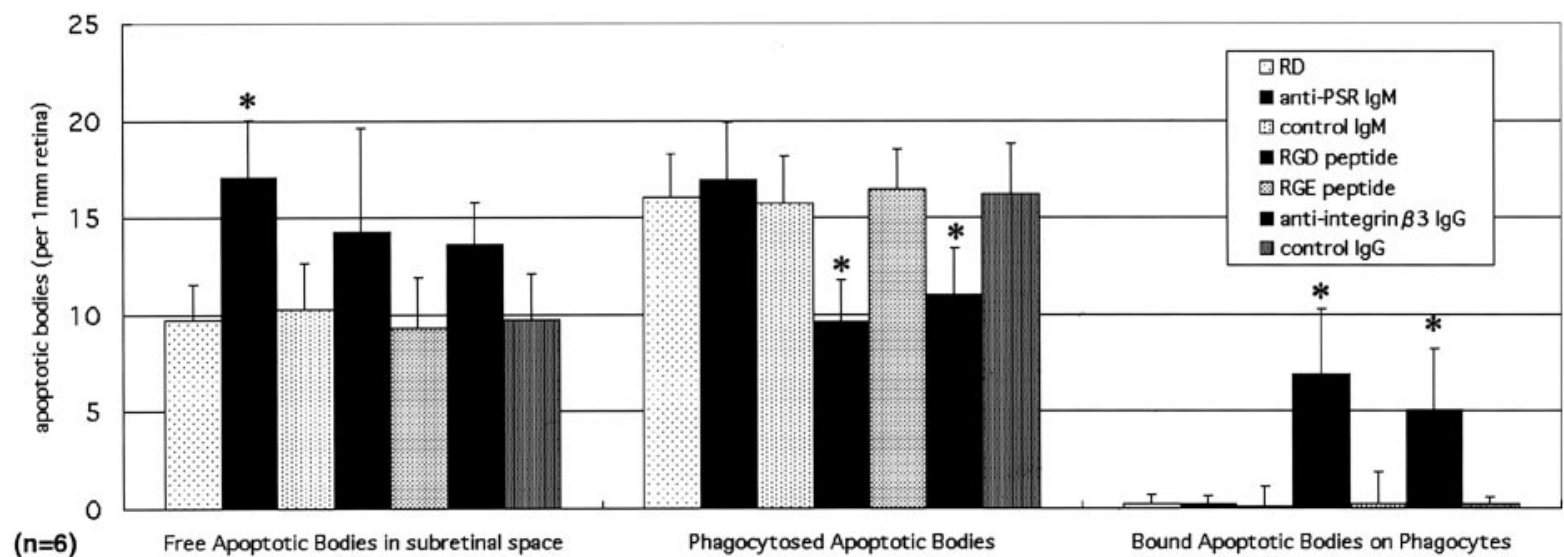

Figure 8. The blockade of the phagocytotic process by inhibiting receptor systems. The function-blocking antibodies and peptides were administered into the subretinal space, respectively. The eyes were harvested 3 days after RD, and then were fixed and embedded in paraffin. The sections were double-stained by TUNEL and propidium iodide. Ten sections for each eye specimen were randomly selected and observed in a masked manner $(n=6)$. The subretinal apoptotic bodies were analyzed as free, phagocytosed, or bound apoptotic bodies, respectively. The anti-PSR IgM increased the free apoptotic bodies in the subretinal space, and decreased the phagocytosed apoptotic bodies $(*, P<0.05)$. The RGD peptide decreased the number of phagocytosed apoptotic bodies, and also caused a dramatic increase in the binding of apoptotic bodies to the macrophages $\left({ }^{*}, P<0.05\right)$. 


\section{Discussion}

The present study clearly demonstrates, for the first time, that apoptotic photoreceptors are selectively eliminated from the original outer nuclear layer of the retina into the subretinal space after RD, and phagocytosed by infiltrated monocyte-derived macrophages. Apparently, these mechanisms allow for the effective clearing of dead photoreceptor cells with minimal tissue inflammation or destruction. The PSR participates in apoptotic photoreceptor recognition, and the integrin $\alpha \vee \beta 3$ participates in internalization of the apoptotic photoreceptors in vivo.

\section{Apoptotic Photoreceptors Eliminated from the Retina in the Late Phase of Apoptosis}

Photoreceptor apoptosis after RD has attracted the interest of neural researchers and has been studied extensively. ${ }^{1,3,18-21,36}$ In this process, photoreceptors develop the characteristic morphological features of apoptosis (initial phase), followed by a clearance process that, schematically, can be subdivided into the subsequent steps of recognition, binding, engulfment, and digestion of apoptotic bodies by phagocytes (late phase). The initial phase has been studied in detail, however, the late phase has not yet been reported. In this study, we clearly showed this late phase of photoreceptor apoptosis after $\mathrm{RD}$ for the first time. Previously, Erickson and colleagues $^{37}$ reported that a loss of photoreceptors occurred both by necrosis and by the migration of photoreceptor into the subretinal space after RD. However, both the origin and mechanism of the late phase still remain unclear. In this study, we demonstrated apoptotic photoreceptors to show chromatin condensation (Figure $2 \mathrm{~B}$ ), electron-dense apoptotic body formation (Figure $2 \mathrm{C}$ ), and electron-dense linear strands (Figure 2D). As apoptotic body formation developed, the electron-dense materials became detectable in the inner and outer segment layer and the subretinal space (Figure 2; E to G). To detect the apoptotic DNA degradation, we examined TUNEL. Although most TUNEL-positive signals were observed in the outer nuclear layer, some signals were observed in the outer segment layer and subretinal space. In this process, we found that AIF, an apoptogenic protein that translocates from the mitochondria to the nucleus after apoptosis, was observed in these electron-dense materials in the outer segment layer, suggesting that these are derived from apoptotic nuclei (Figure 3).

Considering the ultrastructural findings as well as TUNEL and AlF immunohistochemistry, because photoreceptor nuclei are normally located in the outer nuclear layer and are usually not found in the inner and outer segment layers or the subretinal space, these electrondense materials are likely to be displaced apoptotic photoreceptor nuclei from their original location.

During this process, the nuclei translocated from the original nuclear layer into the subretinal space, thus crossing the outer limiting membrane and the outer segment layer. The mechanism through which this active transport of nuclei occurs remains unclear. On theoretical grounds it could be a cell-autonomous process involving an active contraction of the outer part of the cell or it could involve passive processes depending on the specific architecture of the retina. Excreted nuclei from dead photoreceptor cells were effectively phagocytosed by monocyte-derived macrophages that remained confined to the subretinal space. As a result, the neural retina was not invaded by destructive inflammatory cells.

\section{Characterization of Infiltrating Phagocytes after $R D$}

Although detailed ultrastructural studies have been made on infiltrating phagocytes after RD, their characterization and origin have remained elusive. $27,28,38,39$ In this study, we used three antibodies that positively identify monocyte-derived macrophages (ED1), differentiated tissue macrophages (ED2), or RPE (pan cytokeratin), respectively. Those phagocytes that infiltrated into the subretinal space were either ED1 ${ }^{+}$macrophages (88\% of total infiltrated phagocytes) or pan cytokeratin ${ }^{+}$RPEs (12\%) (Figure 4). The ED1 ${ }^{+}$macrophages infiltrated into the subretinal space after RD (Figure 4A), and selectively phagocytosed TUNEL-positive apoptotic debris (Figure $4 C)$. To confirm the origin of these infiltrating phagocytes, we produced chimera mice, namely wild-type C57/B6 mice transplanted with bone marrow cells from GFPtransgenic mice. In such chimera mice, no GFP-positive cells were found in the normal retina (Figure 5A), and GFP-positive cells only infiltrated into the subretinal space after RD (Figure 5, B and C). These results provide compelling evidence that the phagocytes that remove dead photoreceptor nuclei are derived from the transplanted $\mathrm{GFP}^{+}$bone marrow cells, and not from retinal cells such as glial cells. Scanning electron microscopy showed two different types of infiltrating phagocytes in the subretinal space (Figure 6). Considering the results in immunophenotypic studies, these phagocytes were macrophages and RPEs. These macrophages infiltrated from choroidal vessels into the subretinal space beyond the RPE layer (Figure 6C). Surprisingly, the subretinal injection of a neurotrophic factor, BDNF dramatically reduced both photoreceptor apoptosis and macrophage infiltration into the subretinal space (Table 1). The results indicate that monocyte-derived macrophages are selectively called for phagocytotic clearance by apoptotic photoreceptors, not by surgical injury itself.

In addition to macrophages, a small amount of RPE was found to migrate and infiltrate into the subretinal space in the peripheral area of the detached retina (Figure $4 D$ and Figure 6; D to F), as previously described. ${ }^{27,28,38,39}$ RPEs $\left(\mathrm{ED1}^{-} \mathrm{ED}^{-} \mathrm{GFP}^{-}\right.$pancytokeratin ${ }^{+}$) can be readily distinguished from the macrophages by electron microscopy, based on their pigment content, round surface, and the large size (Figure 6; D to F). Scanning electron microscopy also showed that the RPEs migrated from the RPE layer beneath the detached retina (Figure 6F), as described previously. ${ }^{27}$ As a result, the infiltrating phagocytes were macrophages and RPEs, and most infiltrating phagocytes were confirmed to be ED1 ${ }^{+}$, monocyte-de- 
rived macrophages, participating in the clearance of photoreceptors.

\section{Phagocytotic Clearance of Apoptotic Photoreceptors Depends on the PSR for the Recognition and the Integrin $\alpha v \beta 3$ for Internalization}

The phagocytotic processes of apoptosis may include the recognition, binding, internalization, and digestion of apoptotic cells. In our model of RD, the infiltrating macrophages reached apoptotic bodies by elongating filopodia (Figure 7D). The anti-PSR antibody inhibited the selective elongation of filopodia to the apoptotic body, presumably by blocking the interaction between PS on the apoptotic photoreceptors and the PSR on macrophages (Figure 7, B and E). Although macrophages did phagocytose apoptotic bodies, the internalization process is seldom observed under normal conditions. This may be because of the rapid execution of the internalization process. Surprisingly the function-blocking hexapeptide GRGDSP including integrin $\alpha \vee \beta 3$-binding motif RGD, and anti-integrin $\beta 3$ antibody, effectively inhibited this internalization process but did not affect either the recognition or binding processes (Figure 7, C and F, and Figure 8). Scanning electron micrographs clearly demonstrated that many apoptotic bodies were trapped and accumulated on the macrophage surface, yet were not engulfed (Figure 7F). These data demonstrate that effective phagocytotic clearance depends on the PSR for apoptotic photoreceptor recognition, and on the integrin $\alpha \vee \beta 3$ for the internalization of apoptotic photoreceptors in vivo.

In conclusion, the present study revealed the mechanisms through which the selective elimination of apoptotic photoreceptors can be performed in a unique way, which guarantees the conservation of overall retinal tissue architecture after RD. One unresolved issue remains concerning the question as to how apoptotic photoreceptor nuclei translocate from the neural retina to the subretinal space. Further studies are thus called for to clarify this conundrum.

\section{Acknowledgments}

We thank Dr. Shozo Shimokawa, Masao Uehara, and Hiroki Sanui for their financial support; Takaaki Kanemaru in Morphology Core, Kyushu University, for his technical assistance; and Prof. Brian Quinn of Kyushu University for editing the manuscript.

\section{References}

1. Cook B, Lewis GP, Fisher SK, Adler R: Apoptotic photoreceptor degeneration in experimental retinal detachment. Invest Ophthalmol Vis Sci 1995, 36:990-996

2. Witting A, Muller P, Herrmann A, Kettenmann H, Nolte C: Phagocytic clearance of apoptotic neurons by microglia/brain macrophages in vitro: involvement of lectin-, integrin-, and phosphatidylserine-mediated recognition. J Neurochem 2000, 75:1060-1070

3. Hisatomi T, Sakamoto T, Murata T, Yamanaka I, Oshima Y, Hata Y Ishibashi T, Inomata H, Susin SA, Kroemer G: Relocalization of apoptosis-inducing factor in photoreceptor apoptosis induced by retinal detachment in vivo. Am J Pathol 2001, 158:1271-1278

4. Fadok VA, Voelker DR, Campbell PA, Cohen JJ, Bratton DL, Henson PM: Exposure of phosphatidylserine on the surface of apoptotic lymphocytes triggers specific recognition and removal by macrophages. J Immunol 1992, 148:2207-2216

5. Hoffmann PR, deCathelineau AM, Ogden CA, Leverrier Y, Bratton DL, Daleke DL, Ridley AJ, Fadok VA, Henson PM: Phosphatidylserine (PS) induces PS receptor-mediated macropinocytosis and promotes clearance of apoptotic cells. J Cell Biol 2001, 155:649-659

6. Fadok VA, Savill JS, Haslett C, Bratton DL, Doherty DE, Campbell PA, Henson PM: Different populations of macrophages use either the vitronectin receptor or the phosphatidylserine receptor to recognize and remove apoptotic cells. J Immunol 1992, 149:4029-4035

7. Hall SE, Savill JS, Henson PM, Haslett C: Apoptotic neutrophils are phagocytosed by fibroblasts with participation of the fibroblast vitronectin receptor and involvement of a mannose/fucose-specific lectin. J Immunol 1994, 153:3218-3227

8. Finnemann SC, Rodriguez-Boulan E: Macrophage and retinal pigment epithelium phagocytosis: apoptotic cells and photoreceptors compete for alphavbeta3 and alphavbeta5 integrins, and protein kinase $\mathrm{C}$ regulates alphavbeta5 binding and cytoskeletal linkage. $J$ Exp Med 1999, 190:861-874

9. Devitt A, Moffatt OD, Raykundalia C, Capra JD, Simmons DL, Gregory CD: Human CD14 mediates recognition and phagocytosis of apoptotic cells. Nature 1998, 392:505-509

10. Savill J, Hogg N, Ren Y, Haslett C: Thrombospondin cooperates with CD36 and the vitronectin receptor in macrophage recognition of neutrophils undergoing apoptosis. J Clin Invest 1992, 90:1513-1522

11. Ren Y, Silverstein RL, Allen J, Savill J: CD36 gene transfer confers capacity for phagocytosis of cells undergoing apoptosis. J Exp Med 1995, 181:1857-1862

12. Platt N, Suzuki H, Kurihara $Y$, Kodama T, Gordon S: Role for the class A macrophage scavenger receptor in the phagocytosis of apoptotic thymocytes in vitro. Proc Natl Acad Sci USA 1996, 93:12456-12460

13. Luciani MF, Chimini G: The ATP binding cassette transporter ABC1, is required for the engulfment of corpses generated by apoptotic cell death. EMBO J 1996, 15:226-235

14. Botto M, Dell'Agnola C, Bygrave AE, Thompson EM, Cook HT, Petry F, Loos M, Pandolfi PP, Walport MJ: Homozygous C1q deficiency causes glomerulonephritis associated with multiple apoptotic bodies. Nat Genet 1998, 19:56-59

15. Taylor PR, Carugati A, Fadok VA, Cook HT, Andrews M, Carroll MC, Savill JS, Henson PM, Botto M, Walport MJ: A hierarchical role for classical pathway complement proteins in the clearance of apoptotic cells in vivo. J Exp Med 2000, 192:359-366

16. Scott RS, McMahon EJ, Pop SM, Reap EA, Caricchio R, Cohen PL, Earp HS, Matsushima GK: Phagocytosis and clearance of apoptotic cells is mediated by MER. Nature 2001, 411:207-211

17. Maeda $Y$, Shiratsuchi A, Namiki M, Nakanishi Y: Inhibition of sperm production in mice by annexin $V$ microinjected into seminiferous tubules: possible etiology of phagocytic clearance of apoptotic spermatogenic cells and male infertility. Cell Death Differ 2002, 9:742-749

18. Mervin K, Valter K, Maslim J, Lewis G, Fisher S, Stone J: Limiting photoreceptor death and deconstruction during experimental retinal detachment: the value of oxygen supplementation. Am J Ophthalmol 1999, 128:155-164

19. Sakai T, Lewis GP, Linberg KA, Fisher SK: The ability of hyperoxia to limit the effects of experimental detachment in cone-dominated retina. Invest Ophthalmol Vis Sci 2001, 42:3264-3273

20. Lewis GP, Linberg KA, Geller SF, Guerin CJ, Fisher SK: Effects of the neurotrophin brain-derived neurotrophic factor in an experimental model of retinal detachment. Invest Ophthalmol Vis Sci 1999, 40: $1530-1544$

21. Hisatomi T, Sakamoto T, Goto Y, Yamanaka I, Oshima Y, Hata Y, Ishibashi T, Inomata H, Susin SA, Kroemer G: Critical role of photoreceptor apoptosis in functional damage after retinal detachment. Curr Eye Res 2002, 24:161-172

22. Hisatomi T, Sakamoto T, Yamanaka I, Sassa Y, Kubota T, Ueno H, Ohnishi $Y$, Ishibashi T: Photocoagulation-induced retinal gliosis is 
inhibited by systemically expressed soluble TGF- $\beta$ receptor type II via adenovirus mediated gene transfer. Lab Invest 2002, 82:863-870

23. Egensperger R, Maslim J, Bisti S, Hollander H, Stone J: Fate of DNA from retinal cells dying during development: uptake by microglia and macroglia (Muller cells). Brain Res Dev Brain Res 1996, 97:1-8

24. Penfold PL, Provis JM: Cell death in the development of the human retina: phagocytosis of pyknotic and apoptotic bodies by retinal cells. Graefes Arch Clin Exp Ophthalmol 1986, 224:549-553

25. Roque RS, Imperial CJ, Caldwell RB: Microglial cells invade the outer retina as photoreceptors degenerate in Royal College of Surgeons rats. Invest Ophthalmol Vis Sci 1996, 37:196-203

26. Ng TF, Streilein JW: Light-induced migration of retinal microglia into the subretinal space. Invest Ophthalmol Vis Sci 2001, 42:3301-3310

27. Johnson NF, Foulds WS: Observations on the retinal pigment epithelium and retinal macrophages in experimental retinal detachment. $\mathrm{Br} J$ Ophthalmol 1977, 61:564-572

28. Anderson DH, Stern WH, Fisher SK, Erickson PA, Borgula GA: The onset of pigment epithelial proliferation after retinal detachment. Invest Ophthalmol Vis Sci 1981, 21:10-16

29. Fisher SK, Erickson PA, Lewis GP, Anderson DH: Intraretinal proliferation induced by retinal detachment. Invest Ophthalmol Vis Sci 1991 , 32:1739-1748

30. Okabe M, Ikawa M, Kominami K, Nakanishi T, Nishimune Y: 'Green mice' as a source of ubiquitous green cells. FEBS Lett 1997, 407: 313-319

31. Ikawa M, Yamada S, Nakanishi T, Okabe M: 'Green mice' and their potential usage in biological research. FEBS Lett 1998, 430:83-87
32. Susin SA, Lorenzo HK, Zamzami N, Marzo I, Snow BE, Brothers GM, Mangion J, Jacotot E, Costantini P, Loeffler M: Molecular characterization of mitochondrial apoptosis-inducing factor. Nature 1999, 397 441-446

33. Dijkstra CD, Dopp EA, Joling P, Kraal G: The heterogeneity of mononuclear phagocytes in lymphoid organs: distinct macrophage subpopulations in the rat recognized by monoclonal antibodies ED1, ED2 and ED3. Immunology 1985, 54:589-599

34. Austyn JM, Gordon S: F4/80, a monoclonal antibody directed specifically against the mouse macrophage. Eur J Immunol 1981, 11:805815

35. Fadok VA, Bratton DL, Rose DM, Pearson A, Ezekewitz RA, Henson PM: A receptor for phosphatidylserine-specific clearance of apoptotic cells. Nature 2000, 405:85-90

36. Berglin L, Algvere PV, Seregard S: Photoreceptor decay over time and apoptosis in experimental retinal detachment. Graefes Arch Clin Exp Ophthalmol 1997, 235:306-312

37. Erickson PA, Fisher SK, Anderson DH, Stern WH, Borgula GA: Retina detachment in the cat: the outer nuclear and outer plexiform layers. Invest Ophthalmol Vis Sci 1983, 24:927-942

38. Mandelcorn MS, Machemer R, Fineberg E, Hersch SB: Proliferation and metaplasia of intravitreal retinal pigment epithelium cell autotransplants. Am J Ophthalmol 1975, 80:227-237

39. Anderson DH, Stern WH, Fisher SK, Erickson PA, Borgula GA: Retinal detachment in the cat: the pigment epithelial-photoreceptor interface. Invest Ophthalmol Vis Sci 1983, 24:906-926 